\title{
High Resolution Tracking of Cell Membrane Dynamics in Moving Cells: an Electrifying Approach
}

\author{
R.A. Tyson ${ }^{1}$, D.B.A. Epstein ${ }^{2}$, K.I. Anderson ${ }^{3}$ and T. Bretschneider ${ }^{1}$ * \\ ${ }^{1}$ University of Warwick, Warwick Systems Biology Centre, Coventry, UK \\ ${ }^{2}$ University of Warwick, Warwick Mathematics Institute, Coventry, UK \\ ${ }^{3}$ Beatson Institute for Cancer Research, Glasgow, UK
}

\begin{abstract}
Cell motility is an integral part of a diverse set of biological processes. The quest for mathematical models of cell motility has prompted the development of automated approaches for gathering quantitative data on cell morphology, and the distribution of molecular players involved in cell motility. Here we review recent approaches for quantifying cell motility, including automated cell segmentation and tracking. Secondly, we present our own novel method for tracking cell boundaries of moving cells, the Electrostatic Contour Migration Method (ECMM), as an alternative to the generally accepted level set method (LSM). ECMM smoothly tracks regions of the cell boundary over time to compute local membrane displacements using the simple underlying concept of electrostatics. It offers substantial speed increases and reduced computational overheads in comparison to the LSM. We conclude with general considerations regarding boundary tracking in the context of mathematical modelling.
\end{abstract}

Key words: cell movement, tracking, active contour, level set, electrostatics, cytoskeleton, deformable model

AMS subject classification: 92C17, 70Q05

\section{Cartography of the cell}

Cell motility plays an extensive role in many biological processes such as embryogenesis, development, wound healing, and the functioning of the 'search and destroy' cells of the immune system

*Corresponding author. E-mail: T.Bretschneider@warwick.ac.uk 
$[6,28]$. Therefore, the ability of cells to polarise in response to extracellular signals, migrate through extracellular matrices, and dynamically alter in shape are significant focuses for study. Notably, tumor cells are only malignant if capable of migrating [16], while certain parasites depend on migration to invade host tissues. Evidently, the study of cell movement is important for developing treatments for a range of diseases, but also fundamental to understanding the basic principles behind these processes.

Cell motility can be regulated by both internal and external signals that activate intricate signalling cascades resulting in highly dynamic, localised and coordinated remodelling of components of the actin cytoskeleton $[1,38,21,29,28]$. To further elucidate the regulation of cytoskeleton dynamics we must not only identify the structural and regulatory components involved, but critically quantify their spatial-temporal dynamics during movement and their correlation to one another.

\subsection{Imaging moving cells}

Raw data concerning cell movement is typically in the form of time lapse movies, usually involving automated fluorescence microscopy which yields large quantities of complex multichannel data. Cell motility studies often involve the screening of a large number of mutants, or observation of cells in perturbed conditions, and investigation of those that display interesting phenotypes. Obvious problems with this approach are, firstly, that cells affected in critical components of their motile machinery may be completely immobile, useless for quantifying morphology, and, secondly, that differences in phenotypes may be very subtle because of common redundancy of regulatory pathways. Manually screening hundreds of cells for subtle differences is massively time consuming [2]. The need for automated approaches for reproducibly extracting quantitative data from image sequences is therefore plainly apparent $[11,26]$.

Any automated process must address the following requirements: 1) Segmentation of cells from the background of an image sequence to obtain outlines of cells in all frames; 2) Linking cell outlines between frames to allow the tracking of any single cell throughout the image sequence; 3) Mapping of regions of the cell outlines to their corresponding regions in the subsequent frame to allow the tracking of subcellular regions over time; 4) Finally, a methodology is required for measuring fluorescence intensities at subcellular regions, and other locations inside a cell. The main focus of this paper is requirement (3).

Once these requirements have been met, basic quantitative data can be extracted to describe cell movement, such as persistence in direction or chemotactic indices. The DIAS system [34] was one of the first semi-automated systems capable of quantifying aspects of motility. It has been successfully used to analyse the motility behaviour of many cell types [32, 39] and has been updated to perform segmentation of 3D data [33]. Our QuimP software, which will be briefly reviewed in this paper, aims at high resolution measurements of the rates and frequencies of expansion/retraction of specific membrane regions occurring during cell migration. It combines cell shape and motility analysis with fluorescence measurements, enabling the building of detailed spatial-temporal maps correlating a cell's movements with spatial-temporal protein patterns. 


\subsection{Segmentation of moving cells}

To study cell motility, cellular components of the cytoskeleton and its regulators can be stained or tagged with fluorescent probes that, when imaged, appear as intense signals against a black background. The simplest approach to automated segmentation is thresholding, coupled with preprocessing techniques, for example to reduce noise using Gaussian blurring (review [26]). To link objects between frames, for the purpose of tracking, the most probable link is chosen based on attributes such as object displacement, size, or intensity [3], with possible improvements derived from graph theory $[4,37]$.

Thresholding can fail to accurately segment cells due to poor signal to noise, uneven backgrounds, faint edges on cell boundaries, or large intercellular variations in fluorescence [40]. Cells that come into contact are often segmented as single objects because no visible edge exists to distinguish between them. Further problems arise when cells polarise, localising tagged proteins to specific cellular regions, causing uneven fluorescent signals across the cell.

Issues caused by uneven backgrounds can be tackled to an extent, for example by using a local threshold landscape rather than a global threshold [7], but more complex methods have been developed for improved segmentation, in particular deformable models which attempt to match a closed contour to the outline of a cell.

\subsubsection{The deformable model - Kass snake}

Deformable models, or active contours, were first proposed by Kass et al. [20] as a method of guiding a deformable closed contour (or snake) to features of interest within an image, such as edges or lines. A snake is initialised around an object of interest and evolved as a Lagrangian system, in the form of a gradient decent algorithm, which minimises a snake's total energy, $E_{\text {snake }}$

$$
E_{\text {snake }}=E_{\text {int }}+E_{\text {ext }}
$$

where $E_{\text {int }}$ results from internal forces that impose smoothness constraints on the snake (resistance to bending) and $E_{\text {ext }}$ result from external forces derived from the image that pull the snake towards features of interest.

Typically, the total external force is a weighted average computed from the image intensity at any one point $I(x, y)$, allowing the detection of lines, and the image intensity gradient $\nabla I(x, y)$ allowing the detection of edges. As the front evolves the total energy in the system is minimised and the forces acting upon the snake reach equilibrium. The rigidity of the snake imposed by external forces prevents 'leakage' through gaps present in image features, as would occur with seed and growth algorithms. Initialisation of the snake at subsequent frames can be automated by using solutions to previous frames, although this is limited to objects that displace by less then their radius (usually requiring high temporal resolution), therefore providing inherent tracking of cell outlines over the sequence.

Different formulations of the active contour have been proposed to address a snake's poor convergence into concavities [8] and to reduce sensitivity to initialisation caused by the limited capture range of image features. A popular form of active contours is the Gradient Vector Flow 
(GVF) snake that computes a gradient vector potential force field by applying a diffusion process to a traditional potential force field, dispersing the larger magnitude forces out towards homogeneous regions [41]. Similarly, the Force Field Analysis (FFA) snake analyses the properties of the force field to identify and move boundary points that have incorrectly converged [18].

\subsubsection{The deformable model - an implicit approach}

Deformable models can be also used to segment multiple cells. The snake is initialised to encompass multiple cells, and has the functionality to split and merge as objects are encountered, forming multiple closed contours. The ability to split is particularly useful when tracking dividing cells.

An explicit parametric model would require an intricate set of rules for this behaviour, so alternatively an implicit model can be used in which the contour is defined by a zero level set [31]. The level set method (LSM) [27] is a numerical technique for evolving boundaries which inherently supports the merging and splitting of contours, and has the added advantage of being easily extendable to 3D segmentation problems $[14,30]$. We discuss the LSM in the context of boundary tracking in the next section, and provide detailed workings in section 2.1.

Contacting cells may still segment as a single object when using only one deformable model [43]. To avoid such cases Dufour et al. [14] make an initial segmentation of the first frame and associate each segmented object with its own active contour (assuming that all objects are initially spatially isolated). Constraints are imposed that prevent separate active contours overlapping ensuring they only segment their associated cell. The next section discusses approaches to quantify membrane dynamics in detail once a set of cell contours at successive time points has been acquired.

\subsection{High resolution tracking of membrane regions}

We have discussed how to obtain outlines via segmentation, and are now presented with the task of extracting useful information. Obviously, cell outlines provide a means of tracking a cell in terms of its speed, persistence in direction and global shape changes over time, but we also wish to extract detailed quantitative data regarding cell membrane deformations. For example, the speed of protrusions, the rate and frequency of pseudopodia extension, the change in local curvature, and the periodicity of protrusion and retraction. When combined with fluorescence intensity measurements, detailed correlation analyses can be made between spatial-temporal protein patterns and local membrane deformations. For this to be possible, methods to track specific regions of the cell membrane over time must be developed, which is the focus of this paper.

Traditionally, kymographs were used to measure rates of expansion and retraction, for example the membrane activity in human epidermal keratinocytes [22]. This approach is however limited as only partial regions of a cell can be analysed locally. Since active contours are commonly used for segmenting cells, an obvious strategy is to determine membrane deformations globally from a set of successive contours using virtual marker based methods. Virtual markers, defined along the edge of an outline, provide points of reference that can be mapped between successive outlines and hence track specific points over longer periods. Marker displacement provides data on the speed 
at which specific regions move, and the tracking of markers allows for a maintainable set of cell co-ordinates at which data can be sampled from the image, such as the fluorescence intensity of labeled actin. In this manner, marker based methods are of great use in elucidating how protein dynamics underpins membrane deformations and such orchestrates cell motility.

A simple approach would be to define a set of virtual markers on a segmented cell outline at time $T$ and to project those markers onto the outline at time $T+1$ according to normals at marker locations (Figure 1A). However, mapping using normals is only effective when very small deformations are considered. Concave structures and irregular outlines cause virtual marker paths to cross. But this violates the topological requirement that the cell boundary in the image should always be a simple closed curve.
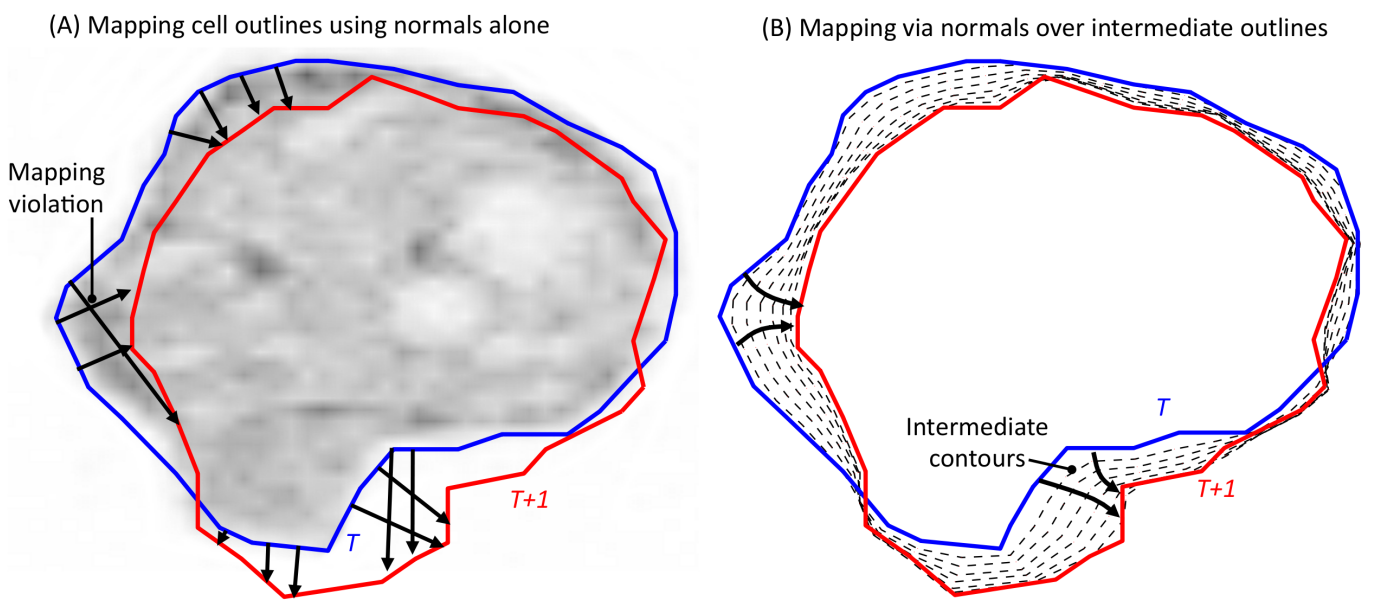

Figure 1: Mapping a cell outline from frame $T$ to $T+1$. A cell is segmented from the image resulting in the outlines $\Gamma_{T}$ (blue) and $\Gamma_{T+1}$ (red). (A) Virtual markers chosen on $\Gamma_{T}$ are mapped to $\Gamma_{T+1}$ by projecting them via normals to $\Gamma_{T}$. Markers often cross paths, effectively causing a twisting of the outline (mapping violation). (B) One solution is to propagate markers using a series of intermediate outlines, $\Gamma_{t}$, over which normals can be followed more reliably (few intermediate outlines are shown here for clarity). This approach of continuous normal propagation can be achieved using the Level Set Method.

Figure 1 illustrates the main problem that is addressed in this paper, namely to develop fast methods that result in a unique mapping of successive contours.

Tsukada et al. [36] developed Edge Evolution Tracking (EET) to study the patterns of RhoGTPases. In EET cell contours in consecutive frames are subdivided into segments defined by the points of intersection and morphological changes are quantified based on the area enclosed by these segments. The use of EET is justified only for very simple deformations.

Currently, the generally accepted method for mapping virtual markers is an implementation of the Level Set Method, previously mentioned as a method of representing an active contour. Machacek and Danuser (2006) use an evolving level set to reconstruct a series of intermediate cell outlines between those resulting from segmentation, and propagate markers via normals to 
the intermediate boundaries (Figure 1B). Markers are still required since the LSM alone does not allow the tracking of specific points on an evolving contour.

The major drawbacks of using the LSM in this context are the computational cost and poor marker distribution (demonstrated in section 2.3). Machacek et al. formulate an alternative approach in the form of an intricate mechanical model of virtual springs that direct the movement of markers, but which works only for deformations that are not too complex. Under large deformations the system of springs is unable to prevent markers crossing over. The mechanical spring model is analogous to using active contours for segmentation, but applied to tracking boundary regions. A similar approach has been used in the QuimP software package, previously developed by us [12], and discussed in more detail in the following section. This paper details the Electrostatic Contour Migration Method (ECMM), a new method for boundary tracking, which supersedes the current tracking in QuimP.

\subsection{QuimP: a framework for quantifying motility and fluorescence}

The QuimP software package [12] forms a framework for automating the measurement of fluorescent intensities of labeled cortical proteins while simultaneously tracking membrane deformations. QuimP (Quantitative imaging of membrane proteins) uses a simple active contour, implemented in ImageJ, to segment cells and define outlines as a chain of nodes (Figure 2). The chain is manually initialised around a cell at $T=0$. Nodes are acted upon by three types of forces; 1) Central forces pull nodes inwards and are required for entering concave regions; 2) Contraction forces between nodes have the overall effect of shrinking the chain by a force vector dependent on the angle between neighbouring nodes; 3) Image forces, proportional to the local contrast of the image, oppose the central force. As the chain approaches the cell boundary, characterised by high contrast, these forces cancel out, neutralising the energy of the snake and halting movement of the contour. The process is repeated at $T+1$, the snake initialised by expanding the solution snake derived at $T$ until it encloses the cell boundary at $T+1$. During shrinkage of the snake, nodes may be added or removed to maintain an average distance between nodes (Figure $2 \mathrm{~F}$ ).

Fluorescence intensity measurements of labeled proteins are taken at regions adjacent to each node. The positions of nodes along the chain are used as co-ordinates for plotting fluorescent intensities allowing visualization of a spatial-temporal map of intensity. For example, Dalous et al. [9] used QuimP to study actin and myo-II relocalization in Dictyostelium discoideum, a unicellular amoeba that plays an important role as a model organism for cell motility [17, 25]. QuimP also outputs numerous statistics useful for analysis including node positions, measured intensities, area changes, cell speed, convexity and centre of mass, as well as computed averages [38].

QuimP is able to successfully measure fluorescent intensities, but is limited in extracting data on local membrane displacements, such as the rate and site of protrusions, because there is no implementation of a membrane region tracking method. An early version of QuimP2 [5] attempts to solve this by assigning nodes unique tracking numbers that are maintained over time. The magnitude of local extensions or retractions are determined by the loss or gain of area between two successive contours that is associated with the displacement of a particular node. A node is defined as extending if it lies within the boundary on the previous frame, else the node is defined 


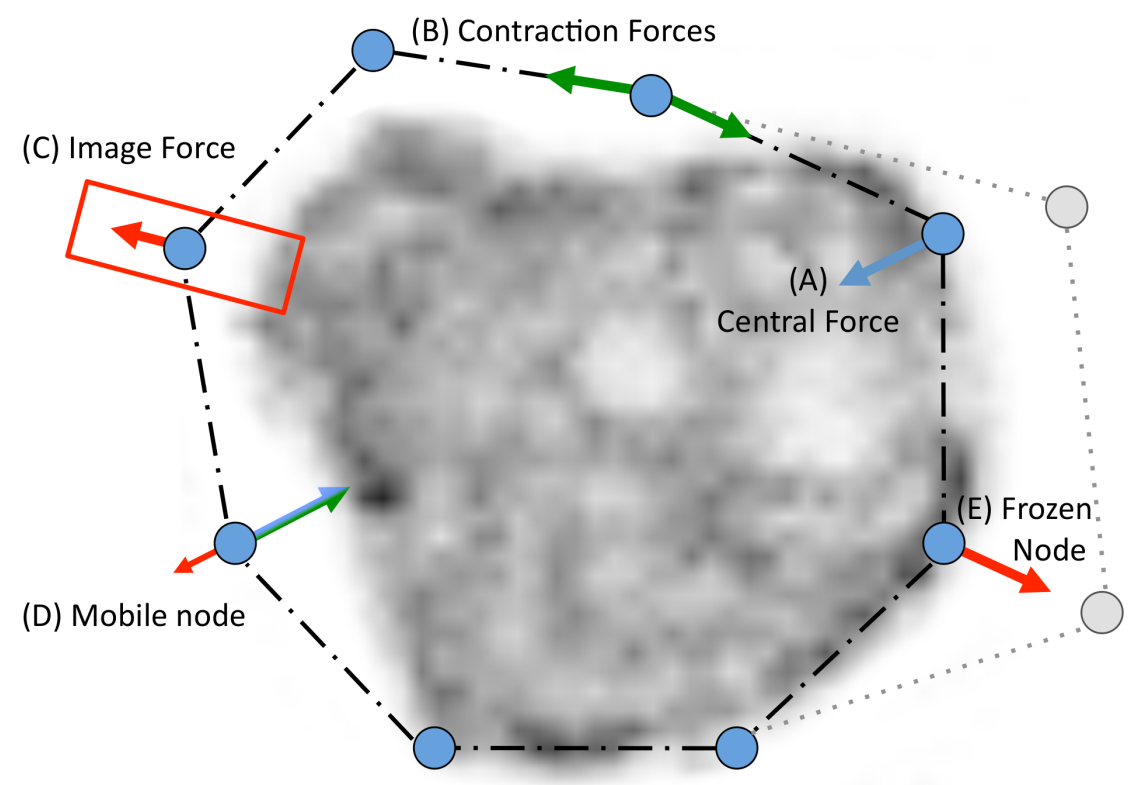

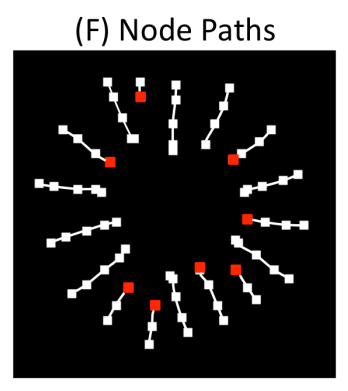

(G) Final Contour

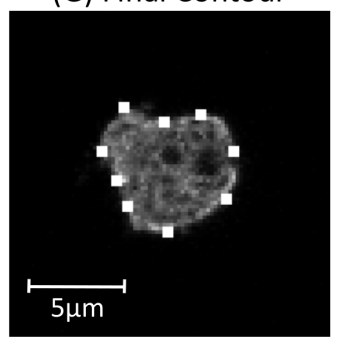

Figure 2: Caricature of outline detection using active contours. The image analysed is that of Dictyostelium discoideum expressing GFP-myosin II at a resolution of 5 pixels $\mu^{-1}$. QuimP's active contour consists of a chain of connected nodes (a reduced number are shown here for simplicity) initialised to encircle a cell of interest. Three types of forces act on each node; (A) Central forces contribute to chain shrinking; (B) Contraction forces between neighbouring nodes shrink the chain and maintain chain integrity; (C) Image forces oppose the shrinking of the chain by a magnitude determined by the local image intensity gradient (red box). When mobile, a node experiences inward forces greater than the opposing image force (D). On reaching the boundary the image force becomes sufficiently large to cancel out the other forces, halting and freezing the node (E). (F) shows the paths of nodes during contraction. Note that nodes can be added or removed (red nodes) to maintain an average distance between neighbours. $(\mathrm{G})$ shows the final position of nodes. QuimP usually makes use of 100 or more nodes to extract high resolution cell outlines.

as retracting. The methodology used by QuimP2 is a fast, one step approach, but the nature of the active contour method means that nodes are continuously being removed and added leading to short node life and discontinuity in tracking nodes. Additionally, the movement of nodes in an active contour poorly represents how real cell boundaries move.

ECMM and LSM both provide virtual marker based tracking of cell outlines independent of the segmentation methodology. In contrast to tracking using active contours, they can be applied at any arbitrary resolution, and markers can be tracked over long periods of time.

\section{The Electrostatic Contour Method}

In this section we present details of the novel Electrostatic Contour Migration for tracking cell boundaries. A LSM approach proposed by Machacek et al. [23] is reviewed and subsequently, an 
elliptical test case forms the bases for comparison of ECMM against the LSM with three different speed functions, which characterise the evolution of a level set. Both methods are implemented in the Matlab programming language (version 2008a).

\subsection{The Electrostatic Contour Migration Method: a novel approach to re- gional tracking}

ECMM is founded on the creation of a fixed electrostatic field formed by evenly placing virtual point charges along the outlines $\Gamma_{T}$ and $\Gamma_{T+1}$, with charges on each outline being opposing. Virtual markers, themselves charged, experience a force determined by the magnitude and direction of the electrostatic field at their location causing them to migrate along field lines.

A marker, positioned at a point $p$ within a field, carrying a single unit of positive charge will experience a force $\overrightarrow{F_{p}}$,

$$
\begin{aligned}
\overrightarrow{F_{p}} & =\sum_{j=1}^{U} C \frac{q_{j}}{r_{j p}^{2.7}} \overrightarrow{e_{j p}}+\sum_{i=1}^{V} C \frac{-q_{i}}{r_{i p}^{2}} \overrightarrow{e_{i p}}, \\
C & =\frac{1}{4 \pi \varepsilon},
\end{aligned}
$$

where $V$ is the number of negative charges, $U$ is the number of positive charges, $C$ is a constant that includes the electrostatic constant $\varepsilon, q$ is the magnitude of a charge creating the electric force, $r$ is the Euclidean distance between $p$ and a charge $q$, and $\vec{e}_{p q}$ is a unit vector from $p$ 's location to $q$ 's. Note that $r$ is raised to the power of 2 for negative charges in accordance with Coulomb's law, whereas for positive charges $r$ is raised to the power of 2.7. This causes the field generated from a positive charge to drop faster with distance: reasons for why we do this are explained later. An example field generated from a negatively charged outline is given in Figure 3A.

The force $\overrightarrow{F_{m}}(p, q)$ experienced by a marker $m$, at position $p$ carrying a charge of magnitude $q$ is given by

$$
\overrightarrow{F_{m}}(p, q)=\overrightarrow{F_{p}} \cdot q
$$

Nodes are migrated through the field by numerically solving the equation

$$
\overrightarrow{p_{t+1}}=p_{t}+\overrightarrow{F_{m}}(p, q) \cdot \Delta t
$$

Cell outlines at $T$ and $T+1$ are overlaid on one another according to their absolute pixel position in the image sequence and their intersection points calculated. Points of intersection compartmentalise the computation of mapping into sectors as shown in Figure 3B. The boundary of a sector consists of an edge $\Upsilon_{T}$ from the cell outline at time $T$, and the corresponding edge $\Upsilon_{T+1}$ from the outline at $T+1$, flanked by intersection points. A sector is designated as expanding if the length of $\Upsilon_{T+1}$ is greater than the length of $\Upsilon_{T}$, or as retracting in the reverse case (Figure $3 \mathrm{C}$ and D). 

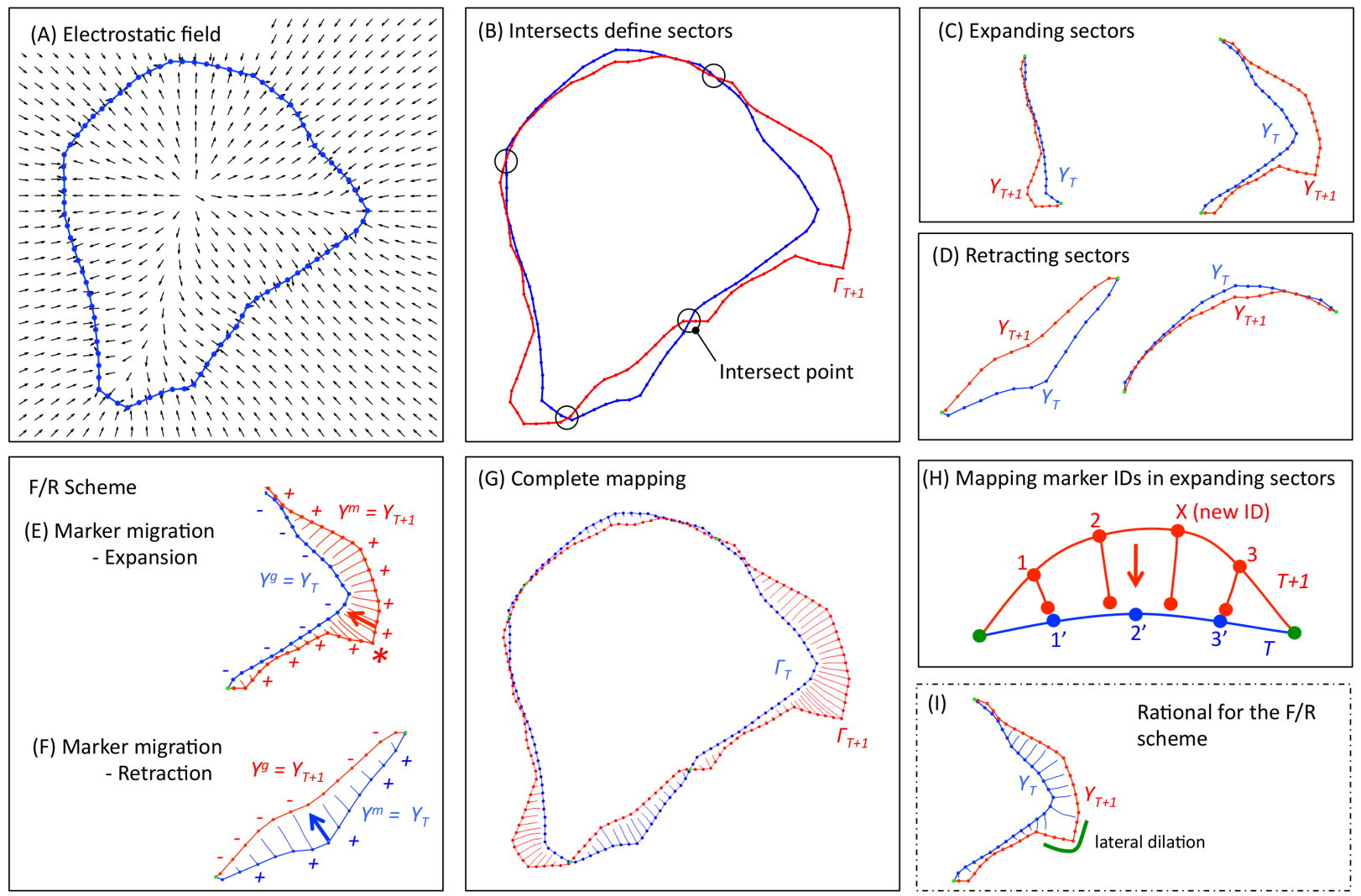

Figure 3: Electrostatic Contour Migration Method (ECMM). (A) shows the electrostatic field, as a vector field, generated by assigning negative point charges along a cell outline (as calculated in Equation 2.1). Vectors represent the magnitude of the force experienced by a single unit of positive charge at points within the field. A positive charge placed within the field will migrate towards the outline. (B) The first step is to overlay the outlines $\Gamma_{T}$ and $\Gamma_{T+1}$ and to compute intersect points. Intersects are used to split the computation into sectors, shown in (C) and (D). Fixing attention on a single sector, we denote its two edges by $\Upsilon_{T} \subset \Gamma_{T}$ and $\Upsilon_{T+1} \subset \Gamma_{T+1}$. Negatively charged markers, held fixed during the interpolation, are placed evenly along the shorter of these two edges, the target edge, denoted by $\Upsilon^{g}$. The longer of the two edges, the migrating edge, denoted by $\Upsilon^{m}$, is provided with evenly placed positively charged markers that move under the influence of the electric field, carrying the edge $\Upsilon^{m}$ with them. (E) illustrates the movement in (C), and includes an * that marks a bulge in the migrating outline. (F) illustrates the movement in (D). Markers, carrying a positive charge, are placed evenly on $\Upsilon^{m}$ and are migrated towards $\Upsilon^{g}$ until within a threshold $d$ of $\Upsilon^{g}$. (G) shows a complete mapping of virtual markers. In the case of retraction, markers retain their unique IDs. In the case of expansion $(\mathrm{H})$ migrated markers (red) compete for the inheritance of marker IDs mapped in the previous time point, based on Euclidean distance. In $(\mathrm{H})$, marker $2^{\prime}$ has been competed for. Markers unable to inherit an ID are assigned the next available integer ID. Finally, (I) shows the rationale for the F/R scheme, without which undesirable lateral dilation occurs. 
Each sector is considered separately for computation; that is to say the electric field for this sector is computed using only the edges $\Upsilon_{T}$ and $\Upsilon_{T+1}$. This is necessary, since expanding and retracting sectors are treated differently, as described in the caption to Figure 3. Furthermore, restricting to these two edges reduces the complexity of the field, thus speeding execution of the code.

The technique is shown in detail in Figure 3 and its caption. Migration of virtual markers is always directed from the longer boundary edge of the sector, termed the migrating edge $\left(\Upsilon^{m}\right)$, to the shorter edge, termed the target edge $\left(\Upsilon^{g}\right)$. This enables us to treat all sectors in a uniform manner, with $\Upsilon^{m}$ moving to $\Upsilon^{g}$. We refer to this as the forward/reverse $(F / R)$ scheme since markers are either mapped forward in time, or backward. The F/R scheme is vital to ECMM, since it avoids marker dilation, which lowers marker resolution in $\Upsilon_{T+1}$. This effect is demonstrated in Figure 3I.

The first step in migrating markers is to place negative point charges evenly along $\Upsilon^{g}$ to attract markers (which carry a positive charge). Positive charges are placed along $\Upsilon^{m}$ to repel markers and prevent them from crossing $\Upsilon^{m}$. Again, this is key to the functioning of ECMM under large deformations, especially in cases where the membrane folds over itself, if we are to avoid the issues encountered by the mechanical model of Machacek et al. (as discussed in section 1.3). As mentioned above, the contribution a positive charge has to the field at distance is less than that of a negative charge. This prevents the migrating edge heavily influencing migrating markers at distance, which could result in markers being compressed together to a point, most problematic when migrating convex edges. For example, overly large positive charges would causes markers located in the bulge in Figure 3E (labeled by an *) to collect in the centre of the bulge.

Markers are then placed on $\Upsilon^{m}$ and allowed to migrate as described by equation (2.4). Positive charges are in fact positioned 2 pixels away from the outer edge of $\Upsilon^{m}$ to ensure markers begin migration in the correct direction, rather than shoot off in the opposite direction. This can happen if a positively charged marker is placed in proximity to the negatively charged edge, but on the wrong side. Obviously, the speed of marker migration is controlled by the magnitude of the charges placed on the various components, and the density of charges on $\Upsilon^{m}$ and $\Upsilon^{g}$. It was necessary to introduce an upper limit on the maximal speed of migration since very large forces can result from point charges in close proximity. When forces become too large our approach becomes numerically unstable and migrating nodes overshoot the target contour. Accuracy is determined by the numerical method (here the inbuilt Matlab ODE23 solver is used), and the speed of migration, both of which can be tuned for the required performance.

Migration is halted when a marker is within a threshold distance, $d$, of the closest point on $\Upsilon^{g}$. The size of $d$ needs to be increased when fewer charges are placed on $\Upsilon^{g}$ because the use of point charges causes the field to become highly variable in close proximity to $\Upsilon^{g}$. Furthermore, the paths of markers can curve as they approach very close to point markers.

Finally, migration is completed by snapping a marker to the closest position on $\Upsilon^{g}$ (moving a marker by roughly the size of $d$ ). Examples of the migration in the two cases, expanding and retracting, are given in Figures $3 \mathrm{E}$ and F. Figure 3G. shows a complete mapping between outlines $\Gamma_{T}$ and $\Gamma_{T+1}$.

To trace virtual markers through time, markers at $T=1$ are labelled, clockwise, by unique integer identifiers $n \ldots N$. To the greatest extent possible, the ID assigned to a marker is not 
changed in subsequent frames. This maximises the period over which a specific marker can be tracked. Details are provided in the caption to Figure 3.

\subsection{Regional tracking using the Level Set Method}

This section describes a level set method for tracking local membrane deformations, as previously implemented by Machacek and Danuser (2006) [23].

The LSM allows the propagation of the cell outline $\Gamma_{T}$ (time $T$ ) onto the outline $\Gamma_{T+1}$. As the boundary is evolved a series of intermediate outlines, $\Gamma_{t}$, are extracted, where $t$ is representative of an evolutionary time step.

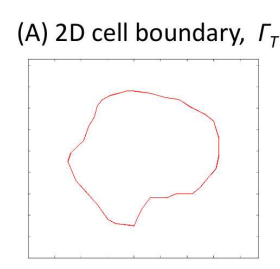

(B) Level set contour map

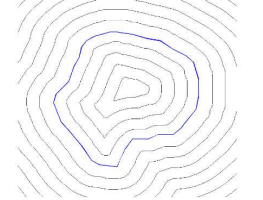

(C) 3D visualization

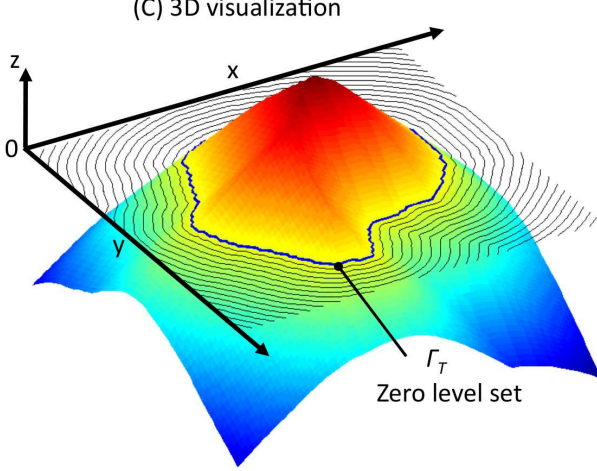

(D) Level set evolution $-\mathrm{z}$ axis displacement

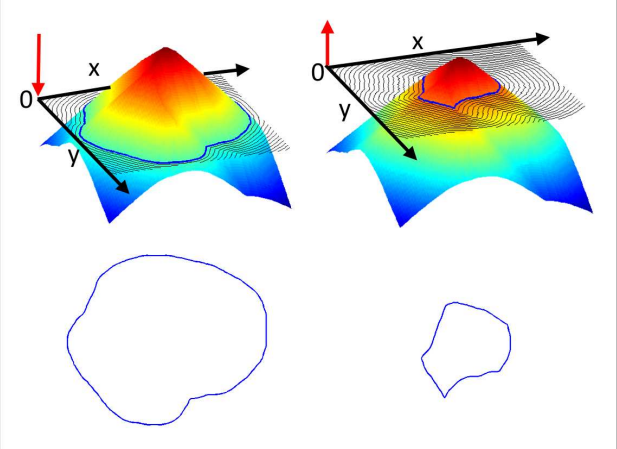

Figure 4: Construction and evolution of the level set. (A) The cell outline at time $T$ is defined on a 2 dimensional grid $\left(\Gamma_{T}(x, y) \in \mathbb{R}^{2}\right)$. (B) $\Gamma_{T}$ is embedded into the level set $\phi_{T}$ by transformation with a signed distance function (the distance a grid point lies from $\Gamma_{T}$ ). Contour lines follow grid points of equal distance from $\Gamma_{T}$. (C) Mapping distance to a third co-ordinate, $z$, allows 3D visualisation of the level set. The outline $\Gamma_{T}$ lies at what is referred to as the zero level set. (D) The level set is displaced in the $z$-axis (either positively or negatively) allowing the extraction of new zero level sets. The outline $\Gamma_{T}$ has been evolved continuously in the normal direction in accordance with the level set surface.

The first step is to define $\Gamma_{T}$ on a two dimensional cartesian grid, $\left(\Gamma_{T}(x, y) \in \mathbb{R}^{2}\right)$, and to transform the grid into a three dimensional surface $\phi_{T}$, referred to as a level set, by applying a signed distance function (Figure 4A, B, C). The distance function assigns $z$ values to $\phi_{T}(x, y)$ equal to the distance between $(x, y)$ and the closest point on $\Gamma_{T}$. Hence, $\Gamma_{T}$ is defined by the zero level set

$$
\Gamma_{T}=\left\{(x, y) \mid \phi_{T}(x, y)=0\right\} .
$$

The outline $\Gamma_{T}$ can be evolved uniformly by the addition of a constant to the signed distance function, effectively displacing $\phi_{T}$ in the $z$ axis (Figure 4D), and extraction of a new zero level set.

Similarly, we can propagate $\phi_{T}$ onto $\phi_{T+1}$ by controlling the speed of evolution across the level set in the evolution equation 


$$
\begin{array}{r}
\frac{\partial \phi(x, y, t)}{\partial t}+F\left(\phi_{t}, t\right)\left|\nabla \phi_{t}(x, y, t)\right|=0, \\
\phi_{(x, y, t=0)}=\phi_{(x, y, T)}
\end{array}
$$

where $F$ is the speed function that controls the speed at which the surface gradient of $\phi_{t}$ is followed (Figure 5). The 'Matlab toolbox implementing Level Set Methods' [35] was used to provide the functionality for evolving level sets. The speed function is critical in influencing the characteristics of the intermediate boundaries, and hence the propagation of normals. The simplest speed function, $F_{d}$ moving the boundary at a speed proportional to its distance from $\Gamma_{T+1}$ is:

$$
F_{d}=\phi_{t}-\phi_{T+1}
$$
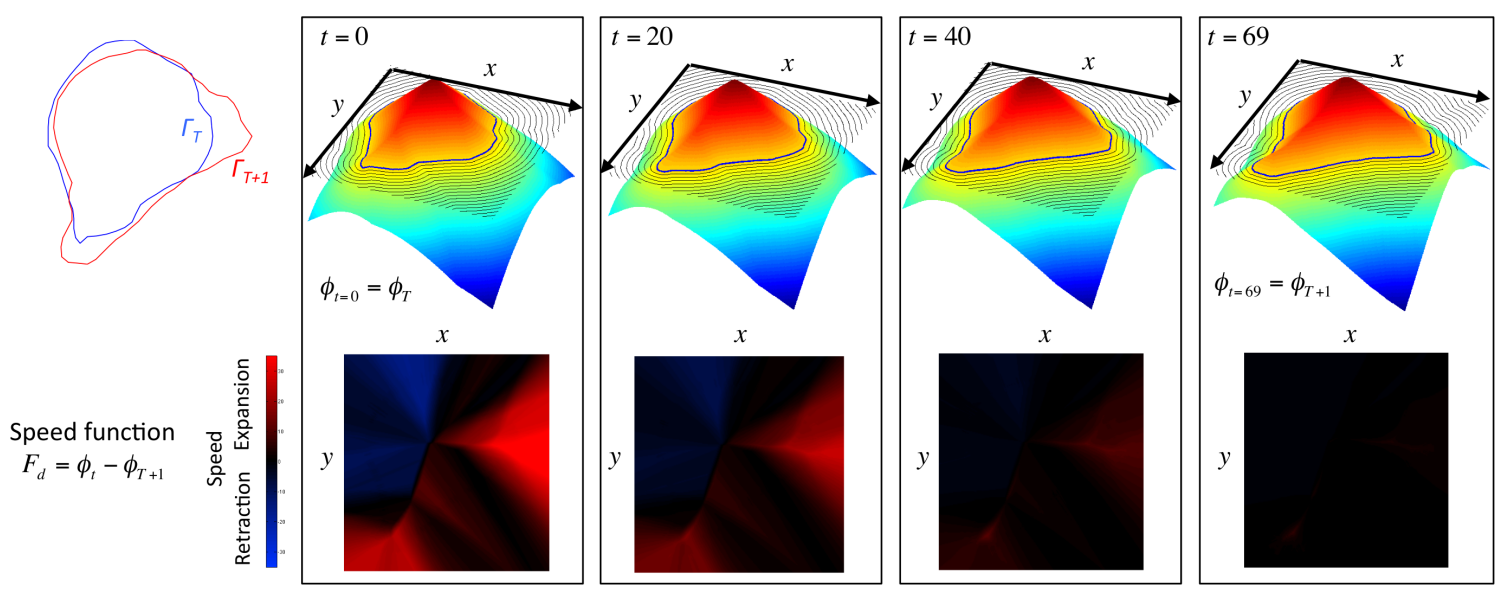

Figure 5: Evolving $\Gamma_{T}$ to converge at $\Gamma_{T+1}$. The level set $\phi_{T}$ is propagated to $\phi_{T+1}$ over time steps $t$ as in the evolution equation (Equation 2.6), until $\phi_{t}=\phi_{T+1}$. Vitally, the speed function $F_{d}$ controls the rate of evolution across the level set and is representative of a distance map between $\phi_{T}$ and $\phi_{T+1}$. As $\phi_{T}$ converges to $\phi_{T+1}$ the speed function is reduced to zero, halting evolution.

During evolution, intermediate contours $\Gamma_{t}$ are extracted as zero level sets, as in equation (2.5). A set of $M$ virtual markers are then propagated to successive intermediate outlines in a direction defined by the normal at the location of the marker on $\Gamma_{t}$ until $\Gamma_{T+1}$ is reached. The resolution of markers, and their propagation, is independent of the computation of the intermediate outlines.

\subsection{Comparison of ECMM and LSM: elliptical test case}

ECMM and LSM are tasked with mapping virtual markers between two overlapping ellipses, one rotated 90 degrees to the other, to simulate both strong expansion in the horizontal direction, and strong retraction in the vertical direction (Figure 6). The problem is computed at a grid size of 
$240 \times 240$ pixels (level sets are embedded in a $240 \times 240$ grid) [Computer spec : $2.16 \mathrm{GHz}$ Intel core 2 duo, 2GB 667MHz DDR2 SDRAM].

Figure 6A. shows the result of mapping using the LSM and speed function given in equation (2.8) (computation time of 21.2 seconds). The evolution of the level set is halted when the absolute size of $F_{d}$ drops below a threshold value. Markers show strong lateral dilation in expanding sectors, leaving expanses of the ellipse at $T+1$ with very low spatial resolution of markers. The opposite problem is observed in retracting sectors with markers congregating centrally to a single point (and overlapping to an extent defined by the accuracy of the numerical solution), undesirably mapping the majority of the boundary to a point.

To reduce dilation and congregation of markers Machacek et al. proposed two additional speed functions.

1. A dampening function (Figure 6B) that slows level set evolution at high speeds

$$
F_{a}=\operatorname{asinh}\left(\phi_{t}-\phi_{T+1}\right)
$$

2. A combination speed function which dampens level set evolution in expanding sectors and determines evolution speed in retracting sectors according to the curvature of the evolving contour (Figure 6C). Highly curved regions evolve faster so that the contour tends towards straightening with the aim of reducing the congregation of the virtual markers,

$$
F_{k}=\left\{\begin{array}{c}
\left(\phi_{t}-\phi_{T+1}\right)>0: \operatorname{asinh}\left(\phi_{t}-\phi_{T+1}\right) \\
\left(\phi_{t}-\phi_{T+1}\right)<0:\left(\phi_{t}-\phi_{T+1}\right)(\epsilon+k)
\end{array},\right.
$$

where $k$ is a second-order finite difference approximation to

$$
k=\frac{\phi_{x x} \phi_{y}^{2}-2 \phi_{y} \phi_{x} \phi_{x y}+\phi_{y y} \phi_{x}^{2}}{\left(\phi_{x}^{2}+\phi_{y}^{2}\right)^{3 / 2}} .
$$

Level set evolution using $F_{a}$ reduces lateral dilation in expanding regions to a certain degree, but the congregating effect on markers in retracting regions is exacerbated (computational time of 23.8 seconds). The speed function $F_{k}$ retains the improvements given by $F_{a}$ and improves marker distribution in retracting regions, but this comes at the expense of slightly increased time till convergence (computational time 31.1 seconds).

Figure 6D. shows the result of mapping using ECMM. The first noteworthy observation is that mappings in retracting and expanding regions are close to identical. This is because the mapping of all deformations are computed as retractions following the F/R scheme. Secondly, the distribution of markers is very uniform throughout the mapping and therefore even marker resolution is achieved along the whole ellipse. Finally, the mapping was computed in a relatively fast time of 1.31 seconds. 


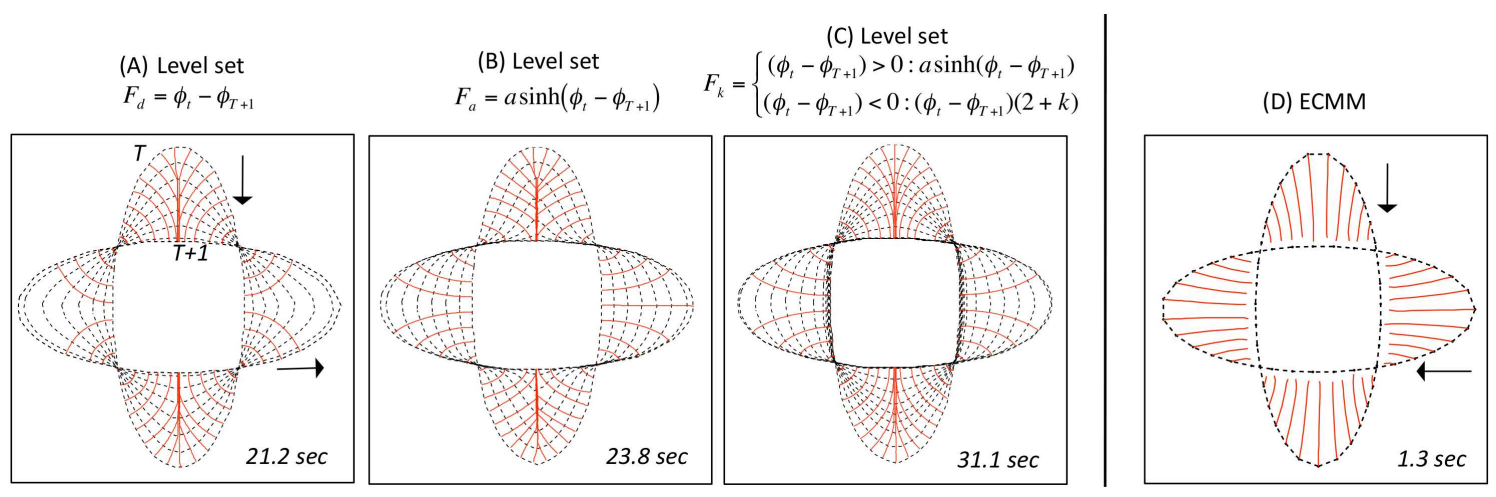

Figure 6: Evaluation of ECMM, and LSM with different speed functions, on an elliptical test case (concept from M.Machacek and G. Danuser (2006), Figure 6). The computational grid size is $240 \times 240$ pixels (marked by bounding boxes). An ellipse at $T$ is migrated to an ellipse at $T+1$ to recreate retracting and expanding sectors. Dashed lines show the progress of the zero level set, while solid red lines show the paths of virtual markers. Arrows indicate the direction in which markers migrate. Timings are provided, in seconds, for computation. (A) The distribution of markers undergoes severe lateral deformation during both retraction and expansion. Markers become dilated during expansion leaving unmapped regions, while congregating centrally during retraction. In this highly compressed region markers may become jumbled, crossing paths. (B) Dilation effects are reduced, but at the expense of increased central compression of markers. (C) The combined speed function reduces the central compression of markers, at the expense of slower convergence. (D) ECMM shows a distribution of markers that is identical during retraction and expansion, and markers remain equidistant with minimal lateral deformation. Satisfactory marker distribution is made possible by ECMM's F/R scheme. ECMM performs the mapping in 1.3 seconds, highlighting its relatively low computational cost.

\section{Discussion}

In this paper we presented ECMM as a novel method for high resolution tracking of cell contours, and compared its performance with current level set method approaches.

The Level Set Method is computationally costly due to the overheads of computing level sets and evolution of continuous contours as apposed to only evolving markers at a spatial resolution defined by the user. The speed of the LSM is defined by the resolution of the grid that defines the level set, the numerical accuracy to which the level set is evolved, and the resolution of markers chosen on the contour. It can be argued that reducing the resolution of the grid improves speed. However, when markers are propagated via normals to intermediate boundaries, then the grid resolution is lowered, and the numerical accuracy of computation of marker paths decreases. This will, in many cases, eventually cause markers to move erratically and their paths to cross.

In ECMM the computational cost is defined by the complexity of the electrostatic field (the number of charges present) and the accuracy of the numerical solution. The complexity of the field has no effect on the validity of marker paths since field lines never cross. Lowering the 
number of charges along the target contour will increase the tendency of markers to curve as they approach, and therefore require a larger threshold, $d$. Additionally, the numerical accuracy can be generally lower, providing even greater speed, since there are no subsequent processes that rely on an accurate solution, such as the propagation of markers in the LSM.

The forward/reverse scheme used to map sectors was a significant milestone in developing ECMM. Not only does it remove the issue of marker dilation during expansion, but the required split into sectors reduces the complexity of the electrostatic field when migrating markers, since only charges within a sector are considered. This is in contrast to LSM where the entire level set is evolved until fully converged.
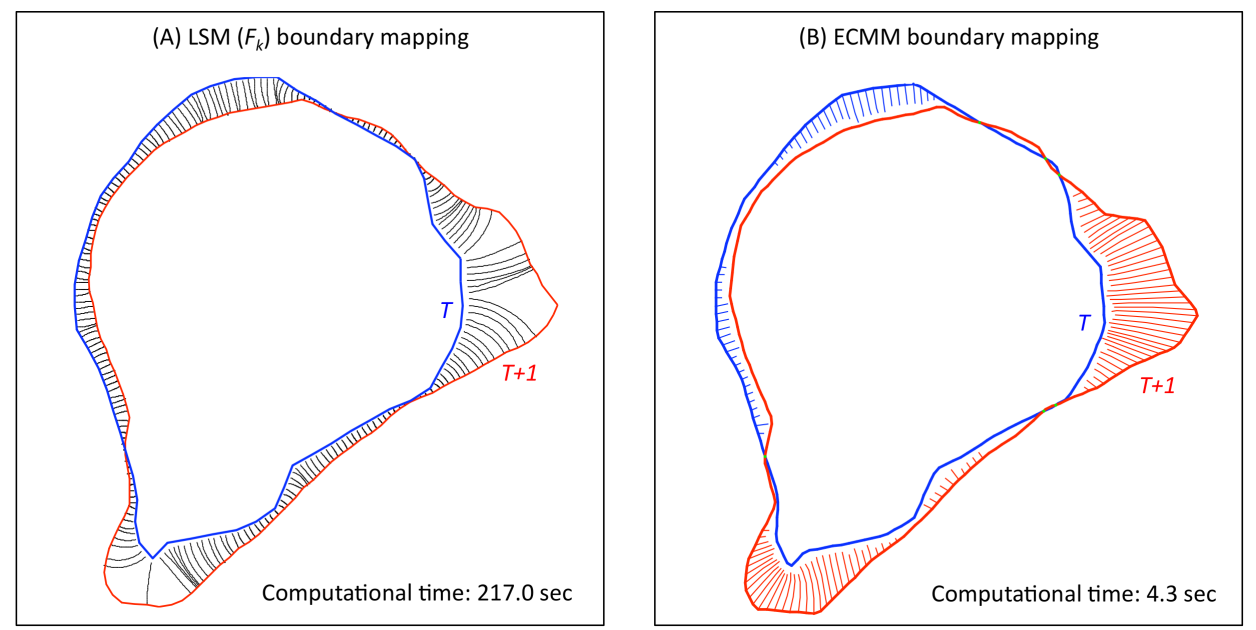

Figure 7: Comparison of boundary mappings on two real cell boundaries. (A) Boundary mapping from $T$ to $T+1$ using the LSM with speed function $\left(F_{k}\right)$. Black lines depict virtual marker paths. Considerable lateral dilation is observed in many parts of the mapping. Computation time: 217.0 seconds. (B) ECMM boundary mapping. Blue lines depict forward mappings and red reverse mappings, as set out in the F/R scheme. Computation time: 4.3 seconds. Marker distribution remains equidistant with minimal lateral deformation.

A comparison of a complete mapping of a real live cell undergoing large deformations is given in Figure 7. ECMM proves to be far faster and does not suffer from marker dilation seen in the mapping computed using the LSM.

ECMM tracks cells in images with the aim of providing quantitative data that can be used for rigorous testing of mathematical models. However it is not a method for modelling actual cell motion and cannot predict the evolution of a cell boundary. This is still the domain of level set methods, which can be simply extended to modelling the mechanical reality of cell membrane protrusion. For example, Yang et al. (2008) [42] developed a viscoelastic model of Dictyostelium parametrised using micropipette aspiration experiments. Deformation of the cell was then simulated by coupling the viscoelastic model to a LSM implementation in which forces acting on the membrane, for example actin polymerisation, can be translated into membrane velocities along the continuous contour. 
Similarly, further work by the Danuser lab (Ji et al. (2008) [19], Machacek et al. (2009) [24]) is extendable to a LSM based mechanical model, although only small fluctuations are considered, so avoiding the pitfalls of LSM. When considering irregular deformations ECMM captures the dynamics of the whole cell better than LSM, a prerequisite for mathematical modelling of cell motility. Table 1 provides an outline of the advantages/disadvantages of ECMM and LSM.

Both LSM and ECMM are methods that help one to interpolate between successive frames of a movie showing cells moving. Both track virtual boundary markers. We wish to stress that the path of a marker is not intended to show the path of some particular molecule or group of molecules on the cell boundary. As the cell moves, the portion of the boundary that you see, what we have called the outline in this paper, will move. Part of the outline may move underneath the cell, part may move to the top of the cell, and part may move into the cell interior. Conversely, the outline in the next frame will come partly from the previous outline, partly from the cell boundary above and below, and partly from inside the cell. In ECMM (and in LSM) each sector contains two points that are fixed during the migration, namely the two vertices of the sector. When a migrating edge happens at about the time of the sudden emergence of a pseudopod, then the fixedness of these two points is clearly not physical and not biological, but an artefact of the method.

Within the literature, no data yet exist describing the true movements of membrane domains during cell membrane protrusion/retraction, especially concerning lateral movement. Validating the movements of virtual markers is therefore currently not feasible. We are currently exploring approaches to elucidate such data, including the use of photobleaching. However, this is experimentally difficult due to the extreme photosensitivity of Dictyostelium cells. Developments in biology and microscopy are expected to yield more information about the true movement of the cell boundary in the not too distant future, and this should have a big effect on studies of the current type.

The two methods presented here are not mutually exclusive and should be considered as complementary to one other.

Keeping track of the virtual markers, although somewhat fictional from the molecular point of view, does nevertheless enable us to make many new significant and biologically meaningful ob-

Table 1: Comparison of advantages/disadvantages of LSM and ECMM

\begin{tabular}{|c|c|}
\hline LSM & ECMM \\
\hline High computational cost & Low computational cost \\
\hline $\begin{array}{l}\text { Marker tracking is stable only for protruding } \\
\text { regions }\end{array}$ & $\begin{array}{l}\text { Marker tracking is stable and robust for both } \\
\text { retracting and expanding regions }\end{array}$ \\
\hline $\begin{array}{l}\text { Poor marker distribution under large mem- } \\
\text { brane deformations }\end{array}$ & Consistently good marker dis \\
\hline $\begin{array}{l}\text { Easily extendable to mechanical modelling of } \\
\text { cell membrane protrusion }\end{array}$ & $\begin{array}{l}\text { In the current form, cannot be used for mod- } \\
\text { elling actual cell membrane movements }\end{array}$ \\
\hline
\end{tabular}


servations. A detailed study of pseudopodia extension, for example, used virtual markers based on active contours to reveal that it is the ratio of actin/myosinII that determines whether pseudopodia extend or retract [5]. ECMM is intended as a superior replacement for further studies.

\subsection{Riemann Mapping Theorem}

We take the basic situation, where the outline curves of a cell in two successive frames of a movie show the movement of the cell, and we wish to interpolate intermediate curves. The two curves may be very nicely related to each other, for example one being only a slight perturbation of the other, with tangent directions not changed much during the perturbation. The underlying biological assumption is that, if we were able to record frames at very high speed, say a millisecond for each frame, then the perturbations would be very slight in this sense. In that case, it is easy to decide how one might move one curve to the other, and both ECMM and LSM would be suitable methods; in fact many other cruder methods would also be applicable. (But ECMM would still be faster than LSM.) Such temporal resolution is very far from attainable with current technology.

As the time lapse between frames increases, interpolation becomes increasingly difficult. Eventually, the two outlines are disjoint from each other, and we have no convincing way to move from one to the other. Even before that, this kind of method will fail. For example, suppose the two curves are as in Figure 8A. Then it is impossible to define sectors on which the above methods might work.

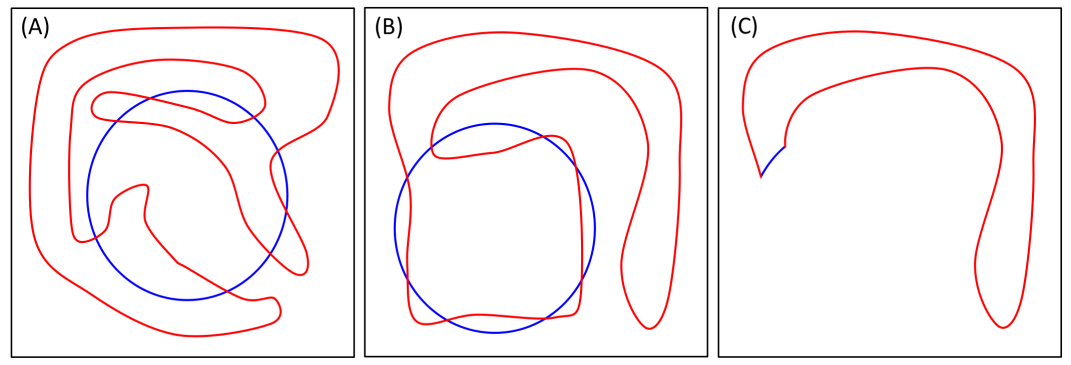

Figure 8: Problematic cell outlines. (A) The intersection of outlines makes defining sectors impossible, causing ECMM and LSM to fail. (B) In cases where sectors can be defined ECMM and LSM may evolve curves that intersect themselves, where $(\mathrm{C})$ is sector from the intersecting outlines in (B). Due to the possibility of these cases we considered using the mathematical approach of Riemann mapping, rather than the physical approach provided by ECMM and LSM.

Now let us look at the situation shown in Figure 8B. where sectors can be defined. We concentrate on the sector show Figure 8C. Here interpolation based on elementary physics principles does not seem to work. Both LSM and ECMM will evolve curves that intersect themselves. The curves here are not realistic biologically; however, realistic biological curves may cause ECMM to fail to produce acceptable marker distributions, and simple examples do fail for LSM (demonstrated by the elliptical test case, section 2.3). For this reason, we considered an approach using mathematical rather than physical principles. 
One of the most important theorems of mathematics is the Riemann Mapping Theorem. To state it, consider the infinite strip $S$ in the plane $S=\{(x, y): 0<y<1\}$. The boundary of $S$ consists of the two lines $y=0$ and $y=1$, together with the two infinite points $+\infty$ and $-\infty$ at the right and left ends of $S$ respectively. The theorem states the following. Let $\Gamma$ be a simple closed curve in the plane and let $U$ be the bounded open region in the plane bounded by $\Gamma$. Let $p$ and $q$ be two distinct points of $\Gamma$. Then there is a homeomorphism $h: S \rightarrow U$ that is conformal (in other words, $h$ is complex differentiable), and which extends continuously to a homeomorphism $h_{\partial}: \partial S \rightarrow \Gamma$, such that $h_{\partial}(-\infty)=p, h_{\partial}(+\infty)=q$. Moreover $h$ is uniquely determined by these conditions, except for the fact that $h$ may be replaced by $h \circ R$, where $R$ is some horizontal translation of $S$.

Provided that the union $\Gamma_{T} \cup \Gamma_{T+1}$ of cell outlines is equal to the boundary of sectors (so the curves in Figure 8A are not allowed), we can apply the Riemann Mapping Theorem to each sector, in such a way that the two intersection points at the two ends of the sector are mapped to $+\infty$ and to $-\infty$. Marker paths are now those paths that correspond under the Riemann Mapping to vertical intervals in $S$. Such vertical intervals are preserved by any horizontal translation $R$ of $S$.

We tried implementing this with Toby Driscoll's SC Toolbox for Matlab [13]. Unsurprisingly, finding the Riemann Mapping is a heavy computation, taking a few hundred seconds instead of the 3 or 4 taken by ECMM. In addition a few of the sectors defeated SC, and the program reported that it was unable to complete the program. It seems as though a certain amount of improvement of Toby Driscoll's Matlab program would be necessary to ensure that it could complete the necessary computations on the sort of examples that come up in biological experiments. In view of the disappointing timings on the vast majority of cases where the computation was successful, it was decided that such an effort would not be justified.

\section{Conclusion}

By developing new methods for tracking virtual markers we aim to aid the study of the mechanisms of cell movement, chemotaxis, signalling to the cytoskeleton, and identification of subtle mutant phenotypes. We detailed ECMM, a novel method for tracking boundary regions, developed as a fast, simplified alternative to the Level Set Method for use in high throughput cell motility analysis tools, such as QuimP. Advantages of ECMM are improved virtual marker distribution while being both simple to implement and computationally cheap.

\section{Acknowledgements}

This research was undertaken at the Systems Biology Doctoral Training Centre, Warwick Systems Biology Centre. Funding provided by the EPSRC and the BBSRC. 


\section{References}

[1] M. Affolter, C. Weijer. Signaling to cytoskeletal dynamics during chemotaxis. Developmental Cell, 9 (2005), No. 1, 19-34.

[2] N. Andrew, R. Insall. Chemotaxis in shallow gradients is mediated independently of PtdIns 3-kinase by biased choices between random protrusions. Nature cell biology, 9 (2007), No. 2, 193-200.

[3] O. Al-Kofahi, R. Radke, S. Goderie, Q. Shen, S. Temple, B. Roysam. Automated cell lineage construction: A rapid method to analyze clonal development established with murine neural progenitor cells. Cell Cycle, 5 (2006), No. 3, 327-335.

[4] B. Bollobas. Modern graph theory. Springer Verlag, 1998.

[5] L. Bosgraaf, P. van Haastert, T. Bretschneider. Analysis of cell movement by simultaneous quantification of local membrane displacement and fluorescent intensities using Quimp2. Cell Motility and the Cytoskeleton, 66 (2009), No. 3, 156-165.

[6] D. Bray. Cell movements: from molecules to motility. Routledge, 2001.

[7] F. Chan, F. Lam, H. Zhu. Adaptive thresholding by variational method. IEEE Transactions on Image Processing, 7 (1998), No.3, 468-473.

[8] L. Cohen. On active contour models and balloons. CVGIP: Image understanding, 53 (1991), No. 2, 211-218.

[9] J. Dalous, E. Burghardt, A. Muller-Taubenberger, F. Bruckert, G. Gerisch, T. Bretschneider. Reversal of cell polarity and actin-myosin cytoskeleton reorganization under mechanical and chemical stimulation. Biophysical journal, 94 (2008), No. 3, 1063-1074.

[10] O. Debeir, I. Camby, R. Kiss, P. Van Ham, C. Decaestecker. A model-based approach for automated in vitro cell tracking and chemotaxis analyses. Cytometry Part A, 60 (2004), 2940 .

[11] C. Decaestecker, O. Debeir, P. Van Ham, R. Kiss. Can anti-migratory drugs be screened in vitro? A review of $2 D$ and $3 D$ assays for the quantitative analysis of cell migration. Medicinal Research Reviews, 27 (2007), No. 2.

[12] D. Dormann, T. Libotte, C. Weijer, T. Bretschneider. Simultaneous quantification of cell motility and protein-membrane-association using active contours. Cell Motil Cytoskeleton, 52 (2002), No. 4, 221-30.

[13] T. Driscoll. The Schwarz-Christoffel Toolbox for MATLAB. Available at: http://www.math.udel.edu/driscoll/software/. (Accessed: 21 Sep. 2009). 
[14] A. Dufour, V. Shinin, S. Tajbakhsh, N. Guillen-Aghion, J. Olivo-Marin, C. Zimmer. Segmenting and tracking fluorescent cells in dynamic 3-D microscopy with coupled active surfaces. IEEE Transactions on Image Proc., 14 (2005), No. 9, 1396-1410.

[15] P. Friedl, B. Weigelin. Interstitial leukocyte migration and immune function. Nature Immunology, 9 (2008), No.9, 960-969.

[16] P. Friedl, Y. Hegerfeldt, M. Tusch. Collective cell migration in morphogenesis and cancer. Int. J. Dev. Biol., 48 (2004), 441-449.

[17] P. Heid, J. Geiger, D. Wessels, E. Voss, D. Soll. Computer-assisted analysis of filopod formation and the role of myosin II heavy chain phosphorylation in Dictyostelium. Journal of cell science, 118 (2005), No. 10, 2225-2237.

[18] Z. Hou, C. Han. Force field analysis snake: an improved parametric active contour model. Pattern Recognition Letters, 26 (2005), No. 5, 513-526.

[19] L. Ji, J. Lim, G. Danuser. Fluctuations of intracellular forces during cell protrusion. Nature cell biology, 10 (2008), No.12, 1393-1400.

[20] M. Kass, A. Witkin, D. Terzopoulos. Snakes: Active contour models. International Journal of Computer Vision, (1988), No. 1, 321-331.

[21] R. Kay, P. Langridge, D. Traynor, O. Hoeller. Changing directions in the study of chemotaxis. Nat. Rev. Mol. Cell Bio, 9 (2008), No. 6, 455-463.

[22] T. Libotte, H. Kaiser, W. Alt, T. Bretschneider. Polarity, protrusion-retraction dynamics and their interplay during keratinocyte cell migration. Experimental Cell Research, 2702001 , No. 2, 129-137.

[23] M. Machacek, G. Danuser. Morphodynamic Profiling of Protrusion Phenotypes Biophysical Journal. Biophysical Soc., 90 (2006), No. 4, 1439-1452.

[24] M. Machacek, L. Hodgson, C. Welch, H. Elliott, O. Pertz, P. Nalbant, A. Abell, G. Johnson, K. Hahn, G. Danuser. Coordination of Rho GTPase activities during cell protrusion. Nature, 461 (2009), No.7260, 99-103.

[25] R. Meili, C. Ellsworth, S. Lee, T. Reddy, H. Ma, R. Firtel. Chemoattractant-mediated transient activation and membrane localization of Akt/PKB is required for efficient chemotaxis to cAMP in Dictyostelium. The EMBO Journal, 18 (1999), No. 8, 2092-2105.

[26] K. Miura. Tracking movement in cell biology. Advances in Biochemical Engineering Biotechnology, 95 (2005), 267-296.

[27] S. Osher, J. Sethian. Fronts propagating with curvature dependent speed: algorithms based on Hamilton-Jacobi formulations. Journal of computational physics, 79 (1988), 12-49. 
[28] A. Ridley, M. Peckham, P. Clark. Cell motility: from molecules to organisms. John Wiley \& Sons Inc, 2004.

[29] A. Ridley, M. Schwartz, K. Burridge, R. Firtel, M. Ginsberg, G. Borisy, J. Parsons, A. Horwitz. Cell Migration: Integrating Signals from Front to Back. Science, 302 (2003), No. 5651, 1704-1709.

[30] A. Sarti, C. Ortiz de Solorzano, S. Lockett, R. Malladi. A geometric model for 3-D confocal image analysis. IEEE Transactions on Biomedical Engineering, 47 (2000), No. 12, 16001609.

[31] J. Sethian. Level set methods and fast marching methods: evolving interfaces in computational geometry, fluid mechanics, computer vision, and materials science. Cambridge Univ Pr, (1999).

[32] D. Shutt, L. Jenkins, E. Carolan, J. Stapleton, K. Daniels, R. Kennedy, D. Soll. T cell syncytia induced by HIV release. T cell chemoattractants: demonstration with a newly developed single cell chemotaxis chamber. J. Cell Sci., 111 (1998), No. 1, 99-109.

[33] D. Soll. Computer-assisted three-dimensional reconstruction and motion analysis of living, crawling cells. Computerized medical imaging and graphics, 23 (1999), No. 1, 3-14.

[34] D. Soll. The use of computers in understanding how animal cells crawl. International review of cytology, 163 (1995), 43-104.

[35] B. Sumengen. A Matlab toolbox implementing Level Set Methods. Available at: http://barissumengen.com/level_set_methods/. (Accessed: 21 Sep. 2009)

[36] Y. Tsukada, K. Aoki, T. Nakamura, Y. Sakumura, M. Matsuda, S. Ishii. Quantification of local morphodynamics and local GTPase activity by edge evolution tracking. PLoS Comp. Bio., 4 (2008), No. 11.

[37] P. Vallotton, A. Ponti, C. Waterman-Storer, E. Salmon, G. Danuser. Recovery, visualization, and analysis of actin and tubulin polymer flow in live cells: a fluorescent speckle microscopy study. Biophysical journal, 85 (2003), No. 2, 1289-1306.

[38] D. Veltman, I. Keizer-Gunnik, P. Van Haastert. Four key signaling pathways mediating chemotaxis in Dictyostelium discoideum. Journal of Cell Biology, 180 (2008), No. 4, 747753.

[39] D. Wessels, E. Voss, N. Von Bergen, R. Burns, J. Stites, D. Soll. A computer-assisted system for reconstructing and interpreting the dynamic three-dimensional relationships of the outer surface, nucleus and pseudopods of crawling cells. Cell motility and the cytoskeleton, 41 (1998), No. 3, 225-246.

[40] K. Wu, D. Gauthier, M. Levine. Live cell image segmentation IEEE Transactions on Biomedical Engineering, 42 (1995), 42, 1-12. 
[41] C. Xu, J. Prince. Snakes, shapes, and gradient vector flow IEEE Transactions on image Processing, IEEE Transactions on image proc., 7 (1998), No. 3, 359-369.

[42] L. Yang, J. Effler, B. Kutscher, S. Sullivan, D. Robinson, P. Iglesias. Modeling cellular deformations using the level set formalism. BMC Systems Biology, 2 (2008), No. 1, 68.

[43] B. Zhang, C. Zimmer, J. Olivo-Marin. Tracking fluorescent cells with coupled geometric active contours. IEEE Inter. Symp. on Biomedical Imaging: Nano to Macro, 1 (2004), 476479. 